\title{
Some Results for Bislant and Semi-Slant Submanifolds of Semi-Riemannian Manifolds
}

\author{
Gülşah AYDIN ŞEKERCi ${ }^{1 *}$ (D) A. Ceylan ÇÖKEN ${ }^{2}$ (D) \\ ${ }^{1}$ Süleyman Demirel University, Department of Mathematics, Isparta, 32260, Turkey \\ ${ }^{2}$ Akdeniz University, Department of Mathematics, Antalya, 07070, Turkey
}

Geliş / Received: 28/03/2021, Kabul / Accepted: 11/06/2021

\begin{abstract}
This study looks into the bislant submanifolds of almost product semi-Riemannian manifolds that are Riemannian, semi-Riemannian and lightlike. We also state the theorems that provide the required conditions for constructing a slant submanifold from a bislant submanifold. Morever, we define the semi-slant submanifolds of locally product semi-Riemannian manifolds and discuss the integrability conditions of distributions, which are invariant or slant, that constitute a semi-slant submanifold.
\end{abstract}

Keywords: Semi-Riemannian; Almost product structure; Nondegenerate metric

\section{Yarı-Riemannian Manifoldların Bislant ve Yarı-Slant Altmanifoldları İçin Bazı Sonuçlar}

Öz

$\mathrm{Bu}$ çalışma hemen hemen çarpım yarı-Riemann manifoldların Riemann, yarı-Riemann ve ışıkbenzer olan bislant altmanifoldlarını incelemektedir. Ayrıca, bir bislant altmanifolddan bir slant altmanifold oluşturmak için gerekli koşulları sağlayan teoremleri ifade ediyoruz. Dahası, lokal çarpım yarı-Riemann manifoldlarının yarıslant altmanifoldlarını tanımlıyor ve yarı-slant bir altmanifold oluşturan değişmez veya slant dağılımların integrallenebilirlik koşullarını tartışıyoruz.

Anahtar Kelimeler: Yarı-Riemann; Hemen hemen çarpım yapısı; Dejenere olmayan metric

\section{Introduction}

Almost product structure $F$ for almost product Riemannian manifolds has been studied for a long time however Yano and Kon (1984)'s paper was the first definition given in the past. Yano and Kon's study was followed with a more detailed paper by Chen (1990). The geometry of slant submanifolds as a generalization of submanifolds for Kaehler manifolds was stated in Chen (1990) which leads new study fields. On almost Riemannian product manifolds; theory of slant and semi-slant submanifolds was presented by Şahin (2006). On locally product manifolds, Li and Liu (2010) studied slant, bislant, semi-slant submanifolds in their paper. Şahin (2008) also stated the slant submanifold for lightlike submanifolds of indefinite Hermitian manifolds. Moreover, Aydın and Çöken (2013) worked the almost product structure on semi-Riemannian manifold similarly to Yano and Kon (1984)'s study. According to that, let $M$ be a $n$-dimensional manifold. $F$ is called an almost product semi-Riemannian structure on $M$ if it is a tensor field of type $(1,1)$ such that $F^{2}=I, F \neq I$ where $I$ is the identity map and $g$ provides the condition $g(F \boldsymbol{X}, \boldsymbol{Y})=g(\boldsymbol{X}, F \boldsymbol{Y})$, for any $\boldsymbol{X}, \boldsymbol{Y} \in \Gamma(T M)$ where $g$ is a semiRiemannian metric tensor. Therefore, $M$ is an almost product semi-Riemannian manifold with almost product semi-Riemannian structure. Also, $M$ is a locally product manifold if $\nabla F=0$ where $\nabla$ is the Levi-Civita connection on $M$ ( $\mathrm{Li}$ and Liu, 2010).

The aim of this study depends on the possible three situations related of semi-Riemannian manifolds which are Riemannian, semi-Riemannian and lightlike submanifolds. It determines 
the slant distributions, bislant submanifolds and semi-slant submanifolds for each mentioned situation. In preliminaries part of this study, some definitions and formulas for almost product semi-Riemannian manifolds are given. On the next part, which also includes the results and discussion, the definitions of slant distributions in these manifolds are introduced. Following these definitions, we describe bislant submanifolds, which are general statements of slant submanifolds, for almost product semi-Riemannian manifold. Furthermore, the necessary and sufficient conditions to do slant submanifold from bislant submanifolds are presented. In addition to these investigations, we also study to evolve some exercises about the bislant submanifolds. Moreover, the semi-slant submanifolds of locally product semi-Riemannian manifolds are defined, and the integrability conditions of invariant or slant distributions that make up a semi-slant submanifold are reviewed.

\section{Preliminaries}

The slant submanifolds on almost product semi- Riemannian manifolds are given by the following three situations since the submanifolds of semi-Riemannian manifold $M$ are Riemannian, semi- Riemannian or lightlike manifolds. Therefore the slant submanifolds are investigated for each situation.

Definition 2.1. Let Riemannian manifold $\bar{M}$ be an immersed submanifold of almost product semi-Riemannian manifold $M$ and $\theta(x)$ be the angle between $F \boldsymbol{X}$ and $\boldsymbol{X}$ for any $x \in \bar{M}, \boldsymbol{X} \in$ $T_{x} \bar{M}$ where $F$ is an almost product structure. If $\theta(x)$ is constant, then it is called the slant angle. Hence, $\bar{M}$ is a slant Riemannian submanifold of $M$ (Aydın and Çöken, 2013).

We have $T M=T \bar{M} \perp T \bar{M}^{\perp}$ since $\bar{M}$ is a Riemannian manifold. For any $\boldsymbol{X} \in \Gamma(T \bar{M})$, we have $\mathrm{F} \boldsymbol{X}=\mathrm{f} \boldsymbol{X}+\mathrm{w} \boldsymbol{X}$ where $\mathrm{f} \boldsymbol{X}$ and $\mathrm{w} \boldsymbol{X}$ are tangent and normal components of vector field $\mathrm{F} \boldsymbol{X}$, respectively. For any $\boldsymbol{V} \in \Gamma\left(T \bar{M}^{\perp}\right)$, we have $\mathrm{F} \boldsymbol{V}=\mathrm{B} \boldsymbol{V}+\mathrm{C} \boldsymbol{V}$ where $B \boldsymbol{V}$ and $\mathrm{C} \boldsymbol{V}$ are tangent and normal components of vector field $\mathrm{F} \boldsymbol{V}$, respectively. Then the Gauss-Weingarten formulas are given by

$$
\begin{aligned}
& \nabla_{\boldsymbol{X}} \boldsymbol{Y}=\bar{\nabla}_{\boldsymbol{X}} \boldsymbol{Y}+h(\boldsymbol{X}, \boldsymbol{Y}) \\
& \nabla_{\boldsymbol{X}} \boldsymbol{V}=-\mathrm{A}_{\boldsymbol{V}} \boldsymbol{X}+\bar{\nabla}^{\perp}{ }_{\boldsymbol{X}} \boldsymbol{V}
\end{aligned}
$$

for any $\boldsymbol{X}, \boldsymbol{Y} \in \Gamma(T \overline{\boldsymbol{M}})$ and $\boldsymbol{V} \in \Gamma\left(T \bar{M}^{\perp}\right)$, where $\bar{\nabla}^{\perp}$ is a connection in the normal bundle, $h$ is a second fundamental form of $\bar{M}$ and $\mathrm{A}_{\boldsymbol{V}}$ is a Weingarten endomorphism associated with $\boldsymbol{V}$ (Li and Liu, 2010).

Let us give the slant submanifold when the submanifold is a semi-Riemannian manifold. Assume that the semi-Riemannian manifold $\bar{M}$ with $n$-dimension is an immersed submanifold of manifold $M$. We show the basis of $T \bar{M}$ by $\left\{\boldsymbol{u}_{1}, \boldsymbol{u}_{2}, \ldots, \boldsymbol{u}_{\boldsymbol{n}}\right\}$. The set of $q$-timelike vector fields is given by $\left\{\boldsymbol{u}_{1}, \boldsymbol{u}_{2}, \ldots, \boldsymbol{u}_{\boldsymbol{q}}\right\}$ and the set of $p$-spacelike vector fields is given by $\left\{\boldsymbol{u}_{q+1}, \boldsymbol{u}_{q+2}, \ldots, \boldsymbol{u}_{n}\right\}$. In this situation, for $q<p, \boldsymbol{f}_{\boldsymbol{i}}$ and $\boldsymbol{f}_{\boldsymbol{i}}^{*}$ are defined by $\boldsymbol{f}_{\boldsymbol{i}}=\frac{\boldsymbol{u}_{\boldsymbol{i}}+\boldsymbol{u}_{\boldsymbol{q}+\boldsymbol{i}}}{\sqrt{2}}$, $\boldsymbol{f}_{i}^{*}=\frac{\boldsymbol{u}_{\boldsymbol{i}}-\boldsymbol{u}_{\boldsymbol{q}+\boldsymbol{i}}}{\sqrt{2}}$ which prove conditions as following:

$$
\begin{gathered}
\mathrm{g}\left(\boldsymbol{f}_{\boldsymbol{i}}, \boldsymbol{f}_{\boldsymbol{j}}\right)=\mathrm{g}\left(\boldsymbol{f}_{\boldsymbol{i}}^{*}, \boldsymbol{f}_{\boldsymbol{j}}^{*}\right)=0, \\
\mathrm{~g}\left(\boldsymbol{f}_{\boldsymbol{i}}, \boldsymbol{f}_{\boldsymbol{j}}^{*}\right)=\delta_{i j}, i, \mathrm{j} \in\{1,2, \ldots, q\} .
\end{gathered}
$$


The basis of $T \bar{M}$ is described by $\left\{\boldsymbol{f}_{\mathbf{1}}, \ldots, \boldsymbol{f}_{\boldsymbol{q}}, \boldsymbol{f}_{\mathbf{1}}^{*}, \ldots, \boldsymbol{f}_{\boldsymbol{q}}^{*}, \boldsymbol{u}_{\mathbf{2 q + 1}}, \ldots, \boldsymbol{u}_{\boldsymbol{p}+\boldsymbol{q}}\right\}$. Let $D$ is a distribution which consists of vector fields $\left\{\boldsymbol{f}_{1}, \ldots, \boldsymbol{f}_{\boldsymbol{q}}\right\}, D^{*}$ is a distribution which consists of vector fields $\left\{\boldsymbol{f}_{\mathbf{1}}^{*}, \ldots, \boldsymbol{f}_{\boldsymbol{q}}^{*}\right\}$ and $D^{\prime}$ is a distribution which consists of vector fields $\left\{\boldsymbol{u}_{\mathbf{2 q}+\mathbf{1}}, \ldots, \boldsymbol{u}_{\boldsymbol{p + q}}\right\}$. So, there exists $T \bar{M}=\left(D \oplus D^{*}\right) \perp D^{\prime}$. Similarly, for $q>p, \boldsymbol{f}_{i}$ and $\boldsymbol{f}_{i}^{*}$ are defined by $\boldsymbol{f}_{\boldsymbol{i}}=\frac{\boldsymbol{u}_{\boldsymbol{i}}+\boldsymbol{u}_{\boldsymbol{q}+\boldsymbol{i}}}{\sqrt{2}}$, $\boldsymbol{f}_{i}^{*}=\frac{\boldsymbol{u}_{i}-\boldsymbol{u}_{\boldsymbol{q}+\boldsymbol{i}}}{\sqrt{2}}$ which prove conditions as following

$$
\begin{gathered}
\mathrm{g}\left(\boldsymbol{f}_{\boldsymbol{i}}, \boldsymbol{f}_{\boldsymbol{j}}\right)=\mathrm{g}\left(\boldsymbol{f}_{\boldsymbol{i}}^{*}, \boldsymbol{f}_{\boldsymbol{j}}^{*}\right)=0, \\
\mathrm{~g}\left(\boldsymbol{f}_{\boldsymbol{i}}, \boldsymbol{f}_{\boldsymbol{j}}^{*}\right)=\delta_{i j}, i, \mathrm{j} \in\{1,2, \ldots, p\} .
\end{gathered}
$$

Thereby, the basis of $T \bar{M}$ is described by $\left\{\boldsymbol{f}_{\mathbf{1}}, \ldots, \boldsymbol{f}_{\boldsymbol{p}}, \boldsymbol{f}_{\mathbf{1}}^{*}, \ldots, \boldsymbol{f}_{\boldsymbol{p}}^{*}, \boldsymbol{u}_{\boldsymbol{p}+\mathbf{1}}, \ldots, \boldsymbol{u}_{\boldsymbol{q}}\right\}$. Let $D$ is a distribution which consists of vector fields $\left\{\boldsymbol{f}_{\mathbf{1}}, \ldots, \boldsymbol{f}_{\boldsymbol{p}}\right\}, D^{*}$ is a distribution which consists of vector fields $\left\{\boldsymbol{f}_{\mathbf{1}}^{*}, \ldots, \boldsymbol{f}_{\boldsymbol{p}}^{*}\right\}$ and $D^{\prime}$ is a distribution which consists of vector fields $\left\{\boldsymbol{u}_{\boldsymbol{p}+\mathbf{1}}, \ldots, \boldsymbol{u}_{\boldsymbol{q}}\right\}$. So, we write again that $T \bar{M}=\left(D \oplus D^{*}\right) \perp D^{\prime}$ (Duggal and Bejancu, 1996).

Definition 2.2. Let semi-Riemannian manifold $\bar{M}$ be an immersed submanifold of almost product semi-Riemannian manifold $M$. For any $x \in \bar{M}, X \in \Gamma\left(D^{\prime}\right), \bar{M}$ is a slant semiRiemannian submanifold of $M$ if and only if the angle $\theta$ between $\boldsymbol{X}$ and $\mathrm{F} \boldsymbol{X}$ is constant where $\mathrm{F}$ is an almost product structure (Aydın and Çöken, 2013).

Now, we can give the slant submanifolds for lightlike submanifolds. Let the lightlike manifold $\bar{M}$ be an immersed submanifold of $M$. We could not write that $T M=T \bar{M} \perp T \bar{M}^{\perp}$ since the manifold $\bar{M}$ is not nondegenerate. In this situation, we benefit from transversal bundle. Then, we have

$$
T M=T \bar{M} \oplus \operatorname{tr}(T \bar{M})
$$

where $\operatorname{tr}(T \bar{M})$ is a transversal bundle. Morever, we have

$$
T \bar{M}=\operatorname{Rad} T \bar{M} \perp S(T \bar{M})
$$

where $\operatorname{Rad} T \bar{M}$ is a radical bundle of $T \bar{M}, S(T \bar{M})$ is a screen bundle of $T \bar{M}$ and is nondegenerate since $\bar{M}$ is a lightlike manifold. Thus, we have

$$
T \bar{M}=\operatorname{Rad} T \bar{M} \perp\left(\mathrm{F} R a d T \bar{M} \oplus \operatorname{Fltr} T \bar{M} \perp D^{\prime \prime}\right)
$$

where if $\operatorname{RadT} \bar{M} \cap \mathrm{FRadT} \bar{M}=\{0\}$ and FRadT $\bar{M}$ is on $S(T \bar{M})$, then $D^{\prime \prime}$ is a complementary distribution to FRadT $\bar{M} \oplus \operatorname{Fltr} T \bar{M}$ on $S(T \bar{M})$. We obtain that

$$
T M=\operatorname{Rad} T \bar{M} \perp S(T \bar{M}) \oplus \operatorname{tr}(T \bar{M}) .
$$

Although $T M$ is a semi-Euclidean space, the metric tensor $\mathrm{g}$ on $\bar{M}$ can be degenerate since $\operatorname{RadT} \bar{M}$ consists of lightlike vector fields. We benefit from lightlike transversal bundle to remove this situation and we obtain

$$
T M=\operatorname{Rad} T \bar{M} \perp S(T \bar{M}) \oplus\left(\operatorname{ltr}(T \bar{M}) \perp D^{\prime}\right)
$$

where $\operatorname{ltr}(T \bar{M})$ is a lightlike transversal bundle, $D^{\prime}$ is a complementary distribution to $\operatorname{lt}(T \bar{M})$ on $\operatorname{tr}(T \bar{M})$ and is a semi-Riemannian distribution. Thus, we describe slant lightlike submanifold as following (Duggal and Bejancu, 1996; Aydın and Çöken, 2013). 
Definition 2.3. Let lightlike manifold $\bar{M}$ be an immersed submanifold of almost product semiRiemannian manifold $M . \bar{M}$ is a slant lightlike submanifold of $M$ if the following conditions provide that;

(i) $\operatorname{FRadT} \bar{M} \cap \operatorname{RadT} \bar{M}=\{0\}$,

(ii) For any $x \in \bar{M}, \boldsymbol{X} \in \Gamma\left(D^{\prime \prime}\right)$, the angle $\theta$ between $\boldsymbol{X}$ and $\mathrm{F} \boldsymbol{X}$ is constant where $D^{\prime \prime}$ is a complementary semi-Riemannian distribution to FRadT $\bar{M} \oplus$ FltrT $\bar{M}$ on $S(T \bar{M})$ and $\quad F X \in$ $\Gamma(T \bar{M})$ (Aydın and Çöken, 2013). (see Şahin (2008) for indefinite Hermitian manifolds)

\section{Bislant and semi-slant submanifolds}

In this section, we study the slant distributions, the bislant submanifolds and semi-slant submanifolds by using the slant submanifolds.

Let $\bar{M}$ be a Riemannian submanifold of almost product semi-Riemannian manifold $M$. We have $T M=T \bar{M} \oplus T \bar{M}^{\perp}$ and we state the slant distribution as following:

Definition 3.1. Let $\bar{M}$ be an immersed Riemannian submanifold of $M$ and $D$ be a distribution on $\bar{M}$. $D$ is a slant distribution on $\bar{M}$ if the angle $\theta(x)$ between $\boldsymbol{X}$ and $\mathrm{F} \boldsymbol{X}$ is constant for any $x \in \bar{M}, \boldsymbol{X} \in D_{x}$.

This situation is different when $\bar{M}$ is semi-Riemannian manifold. Using the decompositions $T M=T \bar{M} \oplus T \bar{M}^{\perp}$ and $T \bar{M}=\left(D \oplus D^{*}\right) \perp D^{\prime}$, we describe the slant distribution as following:

Definition 3.2. Let $\bar{M}$ be an immersed semi-Riemannian submanifold of almost product semiRiemannian manifold M. Assume that the distribution $\bar{D}$ exists on distribution $D^{\prime}$. Then, the distribution $\bar{D}$ is slant distribution on $\bar{M}$ if the angle $\theta(x)$ between $\boldsymbol{X}$ and $\mathrm{F} \boldsymbol{X}$ is constant for any $x \in \bar{M}, \boldsymbol{X} \in \bar{D}_{x}$.

Similarly, we describe the slant distribution from $T \bar{M}=\operatorname{RadT} \bar{M} \perp(\mathrm{F} \operatorname{RadT} \bar{M} \oplus \operatorname{Fltr} T \bar{M} \perp$ $D^{\prime \prime}$ ) when $\bar{M}$ is the lightlike manifold.

Now, we give the definition of bislant submanifolds according to structure of submanifold and we investigate the necessary conditions to obtain the slant submanifold from bislant submanifold.

Definition 3.3. Let $\bar{M}$ be an immersed Riemannian submanifold of almost product semiRiemannian manifold $M$. Then, $\bar{M}$ is called a bislant Riemannian submanifold if it provides the following conditions:

(i) There is orthogonal direct decomposition $T \bar{M}=\left(D_{1} \oplus D_{2}\right)$ where $D_{1}$ and $D_{2}$ are the distributions on $\bar{M}$. 
(ii) $D_{1}$ and $D_{2}$ are the distributions with angles $\theta_{1}$ and $\theta_{2}$, respectively (see Li and Liu (2010) for locally Riemannian manifold).

In this case, we have the following theorem.

Theorem 3.4. Let $\bar{M}$ be an immersed Riemannian submanifold of almost product semiRiemannian manifold $M$ and $\mathrm{g}$ be the metric tensor on $M$. Assume that $\bar{M}$ is a bislant submanifold with slant angle $\theta_{1}=\theta_{2}=\theta . \bar{M}$ is a slant submanifold with slant angle $\theta$ if $\mathrm{g}(\mathrm{F} \boldsymbol{X}, \boldsymbol{Y})=0$ for any $\boldsymbol{X} \in \Gamma\left(D_{1}\right), \boldsymbol{Y} \in \Gamma\left(D_{2}\right)$.

Proof. Assume that $\mathrm{g}(\mathrm{F} \boldsymbol{X}, \boldsymbol{Y})=0$ for any $\boldsymbol{X} \in \Gamma\left(D_{1}\right)$ and $\boldsymbol{Y} \in \Gamma\left(D_{2}\right)$. In this case, we get

$$
\mathrm{g}(\mathrm{f} \boldsymbol{X}+\mathrm{w} \boldsymbol{X}, \boldsymbol{Y})=0
$$

where $\mathrm{f} \boldsymbol{X}$ is the tangent component of $\mathrm{F} \boldsymbol{X}$ and $\mathrm{w} \boldsymbol{X}$ is the normal component of FX. For $\boldsymbol{Y} \in$ $\Gamma\left(D_{2}\right)$, we have $\boldsymbol{Y} \in \Gamma(T \bar{M})$. So, $\mathrm{g}(\mathrm{w} \boldsymbol{X}, \boldsymbol{Y})=0$ is from $\mathrm{w} \boldsymbol{X} \in \Gamma\left(T \bar{M}^{\perp}\right)$. Thus, we find that $\mathrm{g}(\mathrm{f} \boldsymbol{X}, \boldsymbol{Y})=0$. We obtain $\mathrm{f} \boldsymbol{X} \in \Gamma\left(D_{1}\right)$ from $\mathrm{f} \boldsymbol{X} \in \Gamma(T \bar{M})$ and $\boldsymbol{Y} \in \Gamma\left(D_{2}\right)$ since $\mathrm{f} \boldsymbol{X}$ and $\boldsymbol{Y}$ are orthogonal. Also, for any $\boldsymbol{X} \in \Gamma(T \bar{M})$, we get $\boldsymbol{X}=\mathrm{P}_{1} \boldsymbol{X}+\mathrm{P}_{2} \boldsymbol{X}$ where $\mathrm{P}_{1} \boldsymbol{X} \in \Gamma\left(D_{1}\right)$ and $\mathrm{P}_{2} \boldsymbol{X} \in$ $\Gamma\left(D_{2}\right)$. Besides, the angle $\theta_{1}$ between vector fields $\mathrm{fP}_{1} \boldsymbol{X}$ and $\mathrm{FP}_{1} \boldsymbol{X}$ is equal to $\theta$ because $D_{1}$ is the slant distribution with the slant angle $\theta_{1}=\theta$. We obtain

$$
\cos ^{2} \theta=\frac{\left\|\mathrm{fP}_{1} \boldsymbol{X}\right\|^{2}}{\left\|\mathrm{FP}_{1} \boldsymbol{X}\right\|^{2}}
$$

from $\mathrm{FP}_{1} \boldsymbol{X}=\mathrm{fP}_{1} \boldsymbol{X}+\mathrm{wP}_{1} \boldsymbol{X}$. Similarly, we also obtain for the distribution $D_{2}$ that

$$
\cos ^{2} \theta=\frac{\left\|\mathrm{fP}_{2} \boldsymbol{X}\right\|^{2}}{\left\|\mathrm{FP}_{2} \boldsymbol{X}\right\|^{2}} .
$$

So, we have

$$
\begin{aligned}
\frac{\mathrm{g}(\mathrm{f} \boldsymbol{X}, \mathrm{f} \boldsymbol{X})}{\mathrm{g}(\mathrm{F} \boldsymbol{X}, \mathrm{F} \boldsymbol{X})} & =\frac{\mathrm{g}\left(\mathrm{fP}_{1} \boldsymbol{X}+\mathrm{fP}_{2} \boldsymbol{X}, \mathrm{fP}_{1} \boldsymbol{X}+\mathrm{fP}_{2} \boldsymbol{X}\right)}{\mathrm{g}\left(\mathrm{FP}_{1} \boldsymbol{X}+\mathrm{FP}_{2} \boldsymbol{X}, \mathrm{FP}_{1} \boldsymbol{X}+\mathrm{FP}_{2} \boldsymbol{X}\right)} \\
& =\frac{\mathrm{g}\left(\mathrm{fP}_{1} \boldsymbol{X}, \mathrm{fP}_{1} \boldsymbol{X}\right)+\mathrm{g}\left(\mathrm{fP}_{2} \boldsymbol{X}, \mathrm{fP}_{2} \boldsymbol{X}\right)}{\mathrm{g}\left(\mathrm{FP}_{1} \boldsymbol{X}, \mathrm{FP}_{1} \boldsymbol{X}\right)+\mathrm{g}\left(\mathrm{FP}_{2} \boldsymbol{X}, \mathrm{FP}_{2} \boldsymbol{X}\right)} \\
& =\frac{\left\|\mathrm{FP}_{1} \boldsymbol{X}\right\|^{2} \cos ^{2} \theta+\left\|\mathrm{FP}_{2} \boldsymbol{X}\right\|^{2} \cos ^{2} \theta}{\left\|\mathrm{FP}_{1} \boldsymbol{X}\right\|^{2}+\left\|\mathrm{FP}_{2} \boldsymbol{X}\right\|^{2}} \\
& =\cos ^{2} \theta .
\end{aligned}
$$

Thereby, $\bar{M}$ is the slant submanifold with slant angle $\theta$.

The definition of bislant submanifold is different when $\bar{M}$ is the semi-Riemannian submanifold. The reason for this, semi-Riemannian submanifold $\bar{M}$ has $T \bar{M}=\left(D \oplus D^{*}\right) \perp D^{\prime}$ where, for $q<p, D$ is the distribution which is composed by $\left\{\boldsymbol{f}_{\mathbf{1}}, \ldots, \boldsymbol{f}_{\boldsymbol{q}}\right\}, D^{*}$ is the distribution which is composed by $\left\{\boldsymbol{f}_{1}^{*}, \ldots, \boldsymbol{f}_{\boldsymbol{q}}^{*}\right\}, D^{\prime}$ is the distribution which is composed by $\left\{\boldsymbol{u}_{\mathbf{2 q + 1}}, \ldots, \boldsymbol{u}_{\boldsymbol{p}+\boldsymbol{q}}\right\}$ and, for $q>p, D$ is the distribution which is composed by $\left\{\boldsymbol{f}_{\mathbf{1}}, \ldots, \boldsymbol{f}_{\boldsymbol{p}}\right\}, D^{*}$ is the distribution which is composed by $\left\{\boldsymbol{f}_{\mathbf{1}}^{*}, \ldots, \boldsymbol{f}_{\boldsymbol{p}}^{*}\right\}, D^{\prime}$ is the distribution which is composed by $\left\{\boldsymbol{u}_{\boldsymbol{p}+\mathbf{1}}, \ldots, \boldsymbol{u}_{\boldsymbol{q}}\right\}$. 
Definition 3.5. Let $\bar{M}$ be an immersed semi-Riemannian submanifold of almost product semiRiemannian manifold $M$. In this case, $\bar{M}$ is a bislant semi-Riemannian submanifold if it provides the following conditions.

(i) There is $T \bar{M}=\left(D \oplus D^{*}\right) \perp D^{\prime}$ for $\bar{M}$ and $D^{\prime}$ has an orthogonal direct decomposition such that $D^{\prime}=D_{1} \oplus D_{2}$ where $D_{1}$ and $D_{2}$ are the distributions on $\bar{M}$.

(ii) $D_{1}$ and $D_{2}$ are the distributions with slant angle $\theta_{1}$ and $\theta_{2}$, respectively.

Thus, obtaining the slant submanifold from bislant submanifold on semi-Riemannian manifolds is different from Riemannian manifolds.

Theorem 3.6. Let $\bar{M}$ be an immersed semi-Riemannian submanifold of almost product semiRiemannian manifold $M$, g be the metric tensor on $M$ and $\bar{M}$ be the bislant semi-Riemannian submanifold with slant angle $\theta_{1}=\theta_{2}=\theta$. Then, $\bar{M}$ is the slant semi-Riemannian submanifold with slant angle $\theta$ if $\mathrm{g}(\mathrm{F} \boldsymbol{X}, \boldsymbol{Y})=0$ and $\mathrm{f} \boldsymbol{X} \in D^{\prime}$ for any $\boldsymbol{X} \in \Gamma\left(D_{1}\right), \boldsymbol{Y} \in \Gamma\left(D_{2}\right)$.

Proof. We have $T \bar{M}=\left(D \oplus D^{*}\right) \perp D^{\prime}$ since $\bar{M}$ is the semi-Riemannian manifold. Let $\mathrm{P}_{1}, \mathrm{P}_{2}, \mathrm{Q}$ are projection functions on $D, D^{*}, D^{\prime}$, respectively. $Q_{1}, Q_{2}$ are the projection functions on $D_{1}, D_{2}$, respectively, since we have the orthogonal direct decomposition $D^{\prime}=D_{1} \oplus D_{2}$.

Assume that $\mathrm{f} \boldsymbol{X} \in D^{\prime}$ and $\mathrm{g}(\mathrm{F} \boldsymbol{X}, \boldsymbol{Y})=0$ for any $\boldsymbol{X} \in \Gamma\left(D_{1}\right)$ and $\boldsymbol{Y} \in \Gamma\left(D_{2}\right)$. In that case, we get $\mathrm{g}(\mathrm{f} \boldsymbol{X}+\mathrm{w} \boldsymbol{X}, \boldsymbol{Y})=0$

where $\mathrm{f} \boldsymbol{X}$ is the tangent component of $\mathrm{F} \boldsymbol{X}$ and $\mathrm{w} \boldsymbol{X}$ is the normal component of $\mathrm{F} \boldsymbol{X}$. For $\boldsymbol{Y} \in$ $\Gamma\left(D_{2}\right)$, we have $\boldsymbol{Y} \in \Gamma(T \overline{\boldsymbol{M}})$. So, $\mathrm{g}(\mathrm{w} \boldsymbol{X}, \boldsymbol{Y})=0$ is from $\mathrm{w} \boldsymbol{X} \in \Gamma\left(T \bar{M}^{\perp}\right)$. Thus, we find that $\mathrm{g}(\mathrm{f} \boldsymbol{X}, \boldsymbol{Y})=0$ and we have $\mathrm{f} \boldsymbol{X} \in \Gamma\left(D_{1}\right)$ from $\mathrm{f} \boldsymbol{X} \in \Gamma\left(D^{\prime}\right)$.

For any $x \in \bar{M}$ and $X \in \Gamma\left(D^{\prime}\right)$, we will investigate whether $\bar{M}$ is slant submanifold or not. Then, $\mathrm{Q} \boldsymbol{X} \in \Gamma\left(D^{\prime}\right)$ for any $\boldsymbol{X} \in \Gamma(T \bar{M})$. We obtain that $\mathrm{Q} \boldsymbol{X}=\mathrm{Q}_{1} \boldsymbol{X}+\mathrm{Q}_{2} \boldsymbol{X}$ where $\mathrm{Q}_{1} \boldsymbol{X} \in$ $\Gamma\left(D_{1}\right)$ and $\mathrm{Q}_{2} \boldsymbol{X} \in \Gamma\left(D_{2}\right)$. Let $q<p$ where $q$ is the number of timelike vector fields on $T \bar{M}$ and $p$ is the number of spacelike vector fields on $T \bar{M}$. We have

$$
\cos ^{2} \theta=\frac{\left\|\mathrm{fQ}_{1} \boldsymbol{X}\right\|^{2}}{\left\|\mathrm{FQ}_{1} \boldsymbol{X}\right\|^{2}}
$$

since $D_{1}$ is the slant distribution with slant angle $\theta_{1}=\theta$. In addition to, we get

$$
\cos ^{2} \theta=\frac{\left\|\mathrm{fQ}_{2} \boldsymbol{X}\right\|^{2}}{\left\|\mathrm{FQ}_{2} \boldsymbol{X}\right\|^{2}}
$$

since $D_{2}$ is the slant distribution with slant angle $\theta_{2}=\theta$. Then, we obtain that

$$
\begin{aligned}
\frac{\mathrm{g}(\mathrm{fQ} \boldsymbol{X}, \mathrm{fQ} \boldsymbol{X})}{\mathrm{g}(\mathrm{FQ} \boldsymbol{X}, \mathrm{FQ} \boldsymbol{X})} & =\frac{\mathrm{g}\left(\mathrm{fQ}_{1} \boldsymbol{X}+\mathrm{fQ}_{2} \boldsymbol{X}, \mathrm{fQ}_{1} \boldsymbol{X}+\mathrm{fQ}_{2} \boldsymbol{X}\right)}{\mathrm{g}\left(\mathrm{FQ}_{1} \boldsymbol{X}+\mathrm{FQ}_{2} \boldsymbol{X}, \mathrm{FQ}_{1} \boldsymbol{X}+\mathrm{FQ}_{2} \boldsymbol{X}\right)} \\
& =\frac{\mathrm{g}\left(\mathrm{fQ}_{1} \boldsymbol{X}, \mathrm{fQ}_{1} \boldsymbol{X}\right)+\mathrm{g}\left(\mathrm{fQ}_{2} \boldsymbol{X}, \mathrm{fQ}_{2} \boldsymbol{X}\right)}{\mathrm{g}\left(\mathrm{FQ}_{1} \boldsymbol{X}, \mathrm{FQ}_{1} \boldsymbol{X}\right)+\mathrm{g}\left(\mathrm{FQ}_{2} \boldsymbol{X}, \mathrm{FQ}_{2} \boldsymbol{X}\right.} \\
& =\frac{\cos ^{2} \theta \mathrm{g}\left(\mathrm{FQ}_{1} \boldsymbol{X}, \mathrm{FQ}_{1} \boldsymbol{X}\right)+\cos ^{2} \theta \mathrm{g}\left(\mathrm{FQ}_{2} \boldsymbol{X}, \mathrm{FQ}_{2} \boldsymbol{X}\right)}{\mathrm{g}\left(\mathrm{FQ}_{1} \boldsymbol{X}, \mathrm{FQ}_{1} \boldsymbol{X}\right)+\mathrm{g}\left(\mathrm{FQ}_{2} \boldsymbol{X}, \mathrm{FQ}_{2} \boldsymbol{X}\right)}
\end{aligned}
$$


Let $q>p$. We state that

$$
=\cos ^{2} \theta \text {. }
$$

$$
\cosh ^{2} \theta=\frac{\left\|\mathrm{fQ}_{1} \boldsymbol{X}\right\|^{2}}{\left\|\mathrm{FQ}_{1} \boldsymbol{X}\right\|^{2}}
$$

since $D_{1}$ is the slant distribution with slant angle $\theta_{1}=\theta$. In addition to, we have

$$
\cosh ^{2} \theta=\frac{\left\|\mathrm{fQ}_{2} X\right\|^{2}}{\left\|\mathrm{FQ}_{2} \boldsymbol{X}\right\|^{2}}
$$

since $D_{2}$ is the slant distribution with slant angle $\theta_{2}=\theta$. Then, we obtain that

$$
\begin{aligned}
\frac{\mathrm{g}(\mathrm{fQ} \boldsymbol{X}, \mathrm{fQ} \boldsymbol{X})}{\mathrm{g}(\mathrm{FQ} \boldsymbol{X}, \mathrm{FQ} \boldsymbol{X})} & =\frac{\mathrm{g}\left(\mathrm{fQ}_{1} \boldsymbol{X}+\mathrm{fQ}_{2} \boldsymbol{X}, \mathrm{fQ}_{1} \boldsymbol{X}+\mathrm{fQ}_{2} \boldsymbol{X}\right)}{\mathrm{g}\left(\mathrm{FQ}_{1} \boldsymbol{X}+\mathrm{FQ}_{2} \boldsymbol{X}, \mathrm{FQ}_{1} \boldsymbol{X}+\mathrm{FQ}_{2} \boldsymbol{X}\right)} \\
& =\frac{\mathrm{g}\left(\mathrm{fQ}_{1} \boldsymbol{X}, \mathrm{fQ}_{1} \boldsymbol{X}\right)+\mathrm{g}\left(\mathrm{fQ}_{2} \boldsymbol{X}, \mathrm{fQ}_{2} \boldsymbol{X}\right)}{\mathrm{g}\left(\mathrm{FQ}_{1} \boldsymbol{X}, \mathrm{FQ}_{1} \boldsymbol{X}\right)+\mathrm{g}\left(\mathrm{FQ}_{2} \boldsymbol{X}, \mathrm{FQ}_{2} \boldsymbol{X}\right)} \\
& =\frac{\cosh ^{2} \theta \mathrm{g}\left(\mathrm{FQ}_{1} \boldsymbol{X}, \mathrm{FQ}_{1} \boldsymbol{X}\right)+\mathrm{cosh}^{2} \theta \mathrm{g}\left(\mathrm{FQ}_{2} \boldsymbol{X}, \mathrm{FQ}_{2} \boldsymbol{X}\right)}{\mathrm{g}\left(\mathrm{FQ}_{1} \boldsymbol{X}, \mathrm{FQ}_{1} \boldsymbol{X}\right)+\mathrm{g}\left(\mathrm{FQ}_{2} \boldsymbol{X}, \mathrm{FQ}_{2} \boldsymbol{X}\right)} \\
& =\cosh ^{2} \theta .
\end{aligned}
$$

Thereby, $\bar{M}$ is the slant semi-Riemannian submanifold with slant angle $\theta$.

Example 3.7. Let $\bar{M}$ be a submanifold which is immersed of $\mathbb{R}_{2}^{8}=\mathbb{R}_{2}^{4} \times \mathbb{R}^{4}$ and the basis of $T \bar{M}$ is given by the following vector fields:

$\boldsymbol{X}_{1}=(0,1,0,0,0,0,0,0)$,

$X_{2}=(-1,0,1,1,0,0,3,0)$,

$\boldsymbol{X}_{3}=(0,0,1,-1,3 \sqrt{2}, 0,0,0)$,

$\boldsymbol{X}_{4}=(1,0,1,1,0,2 \sqrt{2}, 1,0)$,

$\boldsymbol{X}_{5}=(0,0,0,0,0,0,0,1)$.

Here, the signature of metric tensor $\mathrm{g}$ is given by $(-,-,+,+,+,+,+,+)$ and $F\left(\frac{\partial}{\partial x_{i}}\right)=-\frac{\partial}{\partial x_{i}}$, for $i=1,2,3,4, \quad F\left(\frac{\partial}{\partial x_{j}}\right)=\frac{\partial}{\partial x_{j}}$, for $j=5,6,7,8$ where $\mathrm{F}$ is an almost product semiRiemannian structure. According to that, $p=4$ and $q=1$. So, we obtain

$$
\begin{gathered}
\boldsymbol{f}_{1}=\frac{\boldsymbol{X}_{\mathbf{1}}+\boldsymbol{X}_{\mathbf{5}}}{\sqrt{2}}=\frac{1}{\sqrt{2}}(0,1,0,0,0,0,0,1), \\
\boldsymbol{f}_{1}^{*}=\frac{\boldsymbol{X}_{\mathbf{1}}-\boldsymbol{X}_{\mathbf{5}}}{\sqrt{2}}=\frac{1}{\sqrt{2}}(0,1,0,0,0,0,0,-1) .
\end{gathered}
$$

Then, the distribution $D$ is denoted by $\left\{\boldsymbol{f}_{1}\right\}$ and the distribution $D^{*}$ is denoted by $\left\{\boldsymbol{f}_{1}^{*}\right\}$. Also, we assume that $D_{1}=\operatorname{Sp}\left\{\boldsymbol{X}_{2}, \boldsymbol{X}_{3}\right\}$ and $D_{2}=\operatorname{Sp}\left\{\boldsymbol{X}_{4}\right\}$. In this case, we have

$$
\begin{aligned}
\mathrm{g}\left(\boldsymbol{X}_{2}, \mathrm{~F} \boldsymbol{X}_{2}\right) & =\left\|\boldsymbol{X}_{2}\right\|\left\|\mathrm{F} \boldsymbol{X}_{2}\right\| \cos \beta_{1} \\
8 & =10 \cos \beta_{1} \\
\cos \beta_{1} & =\frac{4}{5}
\end{aligned}
$$

where $\mathrm{F}$ is an almost product semi-Riemannian structure. Morever, we obtain

$$
\begin{aligned}
\mathrm{g}\left(\boldsymbol{X}_{3}, \mathrm{~F} \boldsymbol{X}_{3}\right) & =\left\|\boldsymbol{X}_{3}\right\|\left\|\mathrm{F} \boldsymbol{X}_{3}\right\| \cos \beta_{2} \\
16 & =20 \cos \beta_{2}
\end{aligned}
$$




$$
\cos \beta_{2}=\frac{4}{5}
$$

So, $D_{1}$ is the slant distribution with slant angle $\beta=\cos ^{-1}\left(\frac{4}{5}\right)$. On the other hand, we obtain

$$
\begin{aligned}
\mathrm{g}\left(\boldsymbol{X}_{4}, \mathrm{~F} \boldsymbol{X}_{4}\right) & =\left\|\boldsymbol{X}_{4}\right\|\left\|\mathrm{F} \boldsymbol{X}_{4}\right\| \cos \phi \\
8 & =10 \cos \phi \\
\cos \phi & =\frac{4}{5}
\end{aligned}
$$

Also, $D_{2}$ is the slant distribution with slant angle $\phi=\cos ^{-1}\left(\frac{4}{5}\right) . \bar{M}$ is a bislant submanifold. Besides, $\bar{M}$ is a slant submanifold from $\mathrm{g}\left(\mathrm{F} \boldsymbol{X}_{2}, \boldsymbol{X}_{4}\right)=0$ and $\mathrm{g}\left(\mathrm{FX}_{3}, \boldsymbol{X}_{4}\right)=0$. Also, if we have $D_{2}=\operatorname{Sp}\left\{\boldsymbol{X}_{4}\right\}$ and $\boldsymbol{X}_{4}=(0,1,1, \sqrt{2}, 2 \sqrt{2}, 3,1,0)$, then $\bar{M}$ is not a slant submanifold from $\mathrm{g}\left(\mathrm{FX}_{2}, \boldsymbol{X}_{4}\right) \neq 0$ and $\mathrm{g}\left(\mathrm{FX}_{3}, \boldsymbol{X}_{4}\right) \neq 0$.

Example 3.8. Let $M=\left(\mathbb{R}_{6}^{8}, g\right)=\left(\mathbb{R}_{4}^{4} \times \mathbb{R}_{2}^{4}\right.$,g) be a semi-Riemannian manifold. Assume that the signature of metric tensor $\mathrm{g}$ is given by $(-,-,-,-,-,-,+,+)$ and $\left\{\frac{\partial}{\partial x_{1}}, \frac{\partial}{\partial x_{2}}, \ldots, \frac{\partial}{\partial x_{8}}\right\}$ is the basis of TM. Also, the tangent bundle of $\bar{M}$, which is the submanifold of $\mathbb{R}_{6}^{8}$, is spanned by the following vector fields.

$$
\begin{aligned}
& X_{1}=\frac{\partial}{\partial x_{1}}, \\
& X_{2}=\frac{\partial}{\partial x_{8}}, \\
& X_{3}=\frac{\partial}{\partial x_{2}}+\cos \theta \frac{\partial}{\partial x_{3}}+\sin \theta \frac{\partial}{\partial x_{4}}+2 \frac{\partial}{\partial x_{5}}+\sqrt{2} \frac{\partial}{\partial x_{7}} \\
& X_{4}=2 \frac{\partial}{\partial x_{2}}+\sin \theta \frac{\partial}{\partial x_{3}}-\cos \theta \frac{\partial}{\partial x_{4}}-3 \frac{\partial}{\partial x_{5}}+2 \frac{\partial}{\partial x_{7}} \\
& X_{5}=\frac{\partial}{\partial x_{2}}+\frac{\partial}{\partial x_{3}}+\frac{\partial}{\partial x_{4}}+2 \frac{\partial}{\partial x_{5}}+2 \frac{\partial}{\partial x_{6}}+\sqrt{5} \frac{\partial}{\partial x_{7}}, \\
& X_{6}=\frac{\partial}{\partial x_{2}}-\sqrt{5} \frac{\partial}{\partial x_{5}}+\sqrt{5} \frac{\partial}{\partial x_{6}}+3 \frac{\partial}{\partial x_{7}} .
\end{aligned}
$$

In this situation, $p=1$ and $q=5$. Then, we obtain

$$
\begin{gathered}
f_{1}=\frac{X_{1}+X_{2}}{\sqrt{2}}=\frac{1}{\sqrt{2}}\left(\frac{\partial}{\partial x_{1}}+\frac{\partial}{\partial x_{8}}\right) \\
f_{1}^{*}=\frac{X_{1}-X_{2}}{\sqrt{2}}=\frac{1}{\sqrt{2}}\left(\frac{\partial}{\partial x_{1}}-\frac{\partial}{\partial x_{8}}\right)
\end{gathered}
$$

where $\boldsymbol{f}_{\mathbf{1}}$ and $\boldsymbol{f}_{\mathbf{1}}^{*}$ are the lightlike vector fields. So, the distribution $D$ is denoted by $\left\{\boldsymbol{f}_{\mathbf{1}}\right\}$ and the distribution $D^{*}$ is denoted by $\left\{\boldsymbol{f}_{1}^{*}\right\}$. If $D_{1}$ and $D_{2}$ is given by $D_{1}=\operatorname{Sp}\left\{\boldsymbol{X}_{3}, \boldsymbol{X}_{\mathbf{4}}\right\}$ and $D_{2}=S p\left\{\boldsymbol{X}_{\mathbf{5}}, \boldsymbol{X}_{\mathbf{6}}\right\}$, then we have the orthogonal direct decomposition as given below:

$$
T \bar{M}=\left(D \oplus D^{*}\right) \perp D_{1} \oplus D_{2} .
$$

Now, we will investigate whether $\bar{M}$ is bislant subminifold or not. We define that $F\left(\frac{\partial}{\partial x_{i}}\right)=-\frac{\partial}{\partial x_{i}}$, for $i=1,2,3,4$ and $F\left(\frac{\partial}{\partial x_{j}}\right)=\frac{\partial}{\partial x_{j}}$, for $j=5,6,7,8$ where $F$ is an almost 
product semi-Riemannian structure. Firstly, when we study with distribution $D_{1}$, we need to calculate the angle between $D_{1}$ and $\mathrm{F} \boldsymbol{X}$ for any $\boldsymbol{X} \in \Gamma\left(D_{1}\right)$. Therefore, if we obtain that $\mathrm{F} \boldsymbol{X}_{3}=(0,-1,-\cos \theta,-\sin \theta, 2,0, \sqrt{2}, 0)$ and $\mathrm{F} \boldsymbol{X}_{4}=(0,-2,-\sin \theta, \cos \theta,-3,0,2,0)$, then we have $\mathrm{g}\left(\boldsymbol{X}_{3}, \mathrm{~F} \boldsymbol{X}_{3}\right)=0$ and $\mathrm{g}\left(\boldsymbol{X}_{4}, \mathrm{~F} \boldsymbol{X}_{4}\right)=0$. According to that, we get

$$
\begin{aligned}
\mathrm{g}\left(\boldsymbol{X}_{3}, \mathrm{~F} \boldsymbol{X}_{4}\right) & =\left\|\boldsymbol{X}_{3}\right\|\left\|\mathrm{F} \boldsymbol{X}_{4}\right\| \cosh \alpha_{1} \\
8+2 \sqrt{2} & =2 \sqrt{10} \cosh \alpha_{1} \\
\cosh \alpha_{1} & =\frac{4+\sqrt{2}}{\sqrt{10}}
\end{aligned}
$$

Also, we see that the angle between $\mathrm{F} \boldsymbol{X}_{3}$ and $\boldsymbol{X}_{4}$ is equal to $\alpha_{1}$. So, $D_{1}$ is a slant distribution with slant angle $\alpha_{1}=\cosh ^{-1}\left(\frac{4+\sqrt{2}}{\sqrt{10}}\right)$. Secondly, we study with distribution $D_{2}$. We need to calculate the angle between $D_{2}$ and $\mathrm{F} \boldsymbol{X}$ for any $\boldsymbol{X} \in \Gamma\left(D_{2}\right)$ According to that, if we obtain that $\mathrm{F} \boldsymbol{X}_{5}=(0,-1,-1,-1,2,2, \sqrt{5}, 0)$ and $\mathrm{F} \boldsymbol{X}_{6}=(0,-1,0,0,-\sqrt{5}, \sqrt{5}, 3,0)$, then we have

$$
\begin{aligned}
\mathrm{g}\left(\boldsymbol{X}_{5}, \mathrm{~F} \boldsymbol{X}_{6}\right) & =\left\|\boldsymbol{X}_{5}\right\|\left\|\mathrm{F} \boldsymbol{X}_{6}\right\| \cosh \alpha_{2} \\
3 \sqrt{5}+1 & =\sqrt{6} \sqrt{2} \cosh \alpha_{2} \\
\cosh \alpha_{2} & =\frac{3 \sqrt{5}+1}{2 \sqrt{3}}
\end{aligned}
$$

From here, we say that $D_{2}$ is a slant distribution with slant angle $\alpha_{2}=\cosh ^{-1}\left(\frac{3 \sqrt{5}+1}{2 \sqrt{3}}\right)$. Thus, $\bar{M}$ is a bislant submanifold. Also, $\alpha_{1}$ is not equal to $\alpha_{2}$. In addition to,

$$
\begin{aligned}
\mathrm{g}\left(\boldsymbol{X}_{5}, \mathrm{~F} \boldsymbol{X}_{6}\right)= & \mathrm{g}((0,-1,-\cos \theta,-\sin \theta, 2,0, \sqrt{2}, 0), \\
& (0,1,1,1,2,2, \sqrt{5}, 0)) \\
= & 1+\cos \theta+\sin \theta-4+\sqrt{10} \neq 0
\end{aligned}
$$

Accordingly, $\bar{M}$ is not the slant submanifold.

Now, we investigate the bislant submanifold when $\bar{M}$ is the lightlike submanifold. It is different from the others due to the structure of tangent bundle $T \bar{M}$. We have $T \bar{M}=\operatorname{RadT} \bar{M} \perp$ (FRadT $\bar{M} \oplus \operatorname{Fltr} T \bar{M} \perp D^{\prime \prime}$ ) for lightlike manifold $\bar{M}$ where $D^{\prime \prime}$ is the complementary distribution to $\operatorname{FradT} \bar{M} \oplus \operatorname{Fltr} T \bar{M}$ on $S(T \bar{M})$ and it is semi-Riemannian. Thus, the bislant submanifolds for lightlike submanifold are similar to the bislant semi-Riemannian submanifolds.

Definition 3.9. Let $\bar{M}$ is an immersed lightlike submanifold of almost product semi-Riemannian manifold $M$. Then, $\bar{M}$ is called a bislant lightlike submanifold if it provides the following conditions.

(i) There exists the decomposition $T \bar{M}=\operatorname{RadT} \bar{M} \perp\left(\mathrm{FRadT} \bar{M} \oplus \mathrm{Fltr} T \bar{M} \perp D^{\prime \prime}\right)$ where $D^{\prime \prime}$ has orthogonal direct decomposition $D^{\prime \prime}=D_{1} \oplus D_{2}$ and $D^{\prime \prime}$ is semi-Riemannian. Here, $D_{1}$ and $D_{2}$ are the distributions on $\bar{M}$.

(ii) $D_{1}$ and $D_{2}$ are the distributions with the slant angle $\theta_{1}$ and $\theta_{2}$, respectively. 
Remark 3.10. (i) For ind $(S(T \bar{M}))=\operatorname{nullT} \bar{M}$, the distribution $D^{\prime \prime}$ is extraordinarily Riemannian. Therefore, we need to investigate the bislant notion as similar on bislant Riemannian submanifold.

(ii) For ind $(S(T \bar{M}))>\operatorname{nullT} \bar{M}, D^{\prime \prime}$ is semi-Riemannian and we can state previous theorem as bislant semi-Riemannian submanifold.

Theorem 3.11. Let $\bar{M}$ be an immersed lightlike submanifold of almost product semiRiemannian manifold $M, \mathrm{~g}$ be the metric tensor on $M$ and $\bar{M}$ be a bislant lightlike submanifold with the slant angle $\theta_{1}=\theta_{2}=\theta$. Then, $\bar{M}$ is a slant lightlike manifold with slant angle $\theta$ if $\mathrm{g}(\mathrm{F} \boldsymbol{X}, \boldsymbol{Y})=0$ and $\mathrm{f} \boldsymbol{X} \in \Gamma\left(D^{\prime \prime}\right)$ for any $\boldsymbol{X} \in \Gamma\left(D_{1}\right)$ and $\boldsymbol{Y} \in \Gamma\left(D_{2}\right)$.

Definition 3.12. Let $\bar{M}$ be a Riemannian submanifold of almost product semi-Riemannian manifold M. A semi-slant Riemannian submanifold $\bar{M}$ of $M$ is a submanifold which admits two orthogonal complementary distributions $D_{1}$ and $D_{2}$.

(i) $T \bar{M}=D_{1} \oplus D_{2}$.

(ii)The distribution $D_{1}$ is an invariant distribution, that is $F D_{1}=D_{1}$.

(iii)The distribution $D_{2}$ is a slant distribution with slant angle $\theta \neq 0$ (see Li and Liu (2010) for locally Riemannian manifold).

Theorem 3.13. Let $\bar{M}$ be a Riemannian submanifold of locally product semi-Riemannian manifold M. Then, there exist as followings:

(i) The distribution $D_{1}$ is integrable if and only if

$$
h(\boldsymbol{X}, \mathrm{f} \boldsymbol{Y})=h(\mathrm{f} \boldsymbol{X}, \boldsymbol{Y})
$$

for any $\boldsymbol{X}, \boldsymbol{Y} \in \Gamma\left(D_{1}\right)$ where $h$ is the second fundamental form of $\bar{M}$.

(ii) The distribution $D_{2}$ is integrable if and only if

$$
\mathrm{P}_{1}\left(\nabla_{\boldsymbol{X}} \mathrm{fP}_{2} \mathbf{Y}-\nabla_{\boldsymbol{Y}} \mathrm{fP}_{2} \boldsymbol{X}\right)=\mathrm{P}_{1}\left(A_{\mathrm{w} \boldsymbol{Y}} \boldsymbol{X}-A_{\mathrm{w} \boldsymbol{X}} \boldsymbol{Y}\right)
$$

for any $\boldsymbol{X}, \boldsymbol{Y} \in \Gamma\left(D_{2}\right)$ where $A$ is the shape operator.

Remark 3.14. Above theorem, which is given for semi-slant Riemannian submanifolds of locally product semi-Riemannian manifolds, is demonstrated as in semi slant submanifolds of locally product Riemannian manifolds (see Li and Liu (2010) for locally Riemannian manifold).

Definition 3.15. Let $\bar{M}$ be an immersed semi-Riemannian submanifold of almost product semiRiemannian manifold $M$. In that case, $\bar{M}$ is a semi-slant semi-Riemannian submanifold if it provides the following conditions.

(i) There exists $T \bar{M}=\left(D \oplus D^{*}\right) \perp D^{\prime}$ and $D^{\prime}$ is the orthogonal direct decomposition such that $D^{\prime}=D_{1} \oplus D_{2}$ where $D_{1}$ and $D_{2}$ are the distributions on $\bar{M}$.

(ii) $D_{1}$ is the invariant distribution that is $F D_{1}=D_{1}$

(iii) $D_{2}$ is the slant distribution with slant angle $\theta$. 
Then; let $P_{1}, P_{2}, Q_{1}$ and $Q_{2}$ be projections on $D, D^{*}, D_{1}$ and $D_{2}$, respectively. In this situation, the integrability conditions of distributions could be given by the following theorem.

Theorem 3.16. Let $\bar{M}$ be a semi-Riemannian submanifold of locally product semi-Riemannian manifold $M$. For any $\boldsymbol{X}, \boldsymbol{Y} \in \Gamma\left(D_{1}\right)$,

if the distribution $D_{1}$ is integrable.

$$
h(\boldsymbol{X}, \mathrm{f} \boldsymbol{Y})=h(\mathrm{f} \boldsymbol{X}, \boldsymbol{Y})
$$

Proof. For any $\boldsymbol{X}, \boldsymbol{Y} \in \Gamma\left(D_{1}\right)$, in the Gauss formula given by $\nabla_{\boldsymbol{X}} \boldsymbol{Y}=\bar{\nabla}_{\boldsymbol{X}} \boldsymbol{Y}+h(\boldsymbol{X}, \boldsymbol{Y})$, let us write FY instead of $\boldsymbol{Y}$.

$$
\nabla_{\boldsymbol{X}} \mathrm{F} \boldsymbol{Y}=\bar{\nabla}_{\boldsymbol{X}} F \boldsymbol{Y}+h(\boldsymbol{X}, \mathrm{F} \boldsymbol{Y})
$$

$\nabla \mathrm{F}=0$ since $\bar{M}$ is locally product semi-Riemannian manifold, so $\nabla_{\boldsymbol{X}} \mathrm{F} \boldsymbol{Y}=\mathrm{F} \nabla_{\boldsymbol{X}} \boldsymbol{Y}$. From this equation, we have

$$
\bar{\nabla}_{\boldsymbol{X}} \mathrm{F} \boldsymbol{Y}+h(\boldsymbol{X}, \mathrm{F} \boldsymbol{Y})=\mathrm{F} \bar{\nabla}_{\boldsymbol{X}} \boldsymbol{Y}+\mathrm{F} h(\boldsymbol{X}, \boldsymbol{Y})
$$

When we separate this equation to the tangential and normal components, there are

$$
\begin{aligned}
& \bar{\nabla}_{\boldsymbol{X}}(\mathrm{f} \boldsymbol{Y}+\mathrm{w} \boldsymbol{Y})+h(\boldsymbol{X}, \mathrm{F} \boldsymbol{Y})=\overline{\mathrm{f}}_{\boldsymbol{X}} \boldsymbol{Y}+\mathrm{w} \bar{\nabla}_{\boldsymbol{X}} \boldsymbol{Y}+B h(\boldsymbol{X}, \boldsymbol{Y})+C h(\boldsymbol{X}, \boldsymbol{Y}) \\
& \bar{\nabla}_{\boldsymbol{X}} \mathrm{f} \boldsymbol{Y}+\bar{\nabla}_{\boldsymbol{X}} \mathrm{w} \boldsymbol{Y}+h(\boldsymbol{X}, \mathrm{F} \boldsymbol{Y})=\overline{\mathrm{f}}_{\boldsymbol{X}} \boldsymbol{Y}+\mathrm{w} \bar{\nabla}_{\boldsymbol{X}} \boldsymbol{Y}+B h(\boldsymbol{X}, \boldsymbol{Y})+C h(\boldsymbol{X}, \boldsymbol{Y})
\end{aligned}
$$

Since the distribution $D_{1}$ is invariant and $\boldsymbol{X}, \boldsymbol{Y} \in \Gamma\left(D_{1}\right)$, we have $\mathrm{w} \boldsymbol{Y}=0$.

$$
\bar{\nabla}_{\boldsymbol{X}} \mathrm{f} \boldsymbol{Y}+h(\boldsymbol{X}, \mathrm{F} \boldsymbol{Y})=f \bar{\nabla}_{\boldsymbol{X}} \boldsymbol{Y}+\mathrm{w} \bar{\nabla}_{\boldsymbol{X}} \boldsymbol{Y}+B h(\boldsymbol{X}, \boldsymbol{Y})+C h(\boldsymbol{X}, \boldsymbol{Y})
$$

So, we can easily see that

$$
\mathrm{w} \nabla_{\boldsymbol{X}} \boldsymbol{Y}=h(\boldsymbol{X}, \mathrm{F} \boldsymbol{Y})-\operatorname{Ch}(\boldsymbol{X}, \boldsymbol{Y})
$$

By replacing $\boldsymbol{X}$ and $\boldsymbol{Y}$,

$$
\mathrm{w}\left(\nabla_{\boldsymbol{X}} \boldsymbol{Y}-\nabla_{\boldsymbol{Y}} \boldsymbol{X}\right)=h(\boldsymbol{X}, \mathrm{F} \boldsymbol{Y})-h(\boldsymbol{Y}, \mathrm{F} \boldsymbol{X})
$$

If $D_{1}$ is integrable, then $[\boldsymbol{X}, \boldsymbol{Y}] \in \Gamma\left(D_{1}\right)$ and from here, $\mathrm{w}[\boldsymbol{X}, \boldsymbol{Y}]=0$. So, the desired equation is obtained.

However, the opposite of the above theorem is not always true. It is given by the following result.

Conclusion 3.17. Let $\bar{M}$ be a semi-Riemannian submanifold of locally product semiRiemannian manifold $M$. For any $\boldsymbol{X}, \boldsymbol{Y} \in \Gamma\left(D_{1}\right)$,

$$
h(\boldsymbol{X}, \mathrm{f} \boldsymbol{Y})=h(\mathrm{f} \boldsymbol{X}, \boldsymbol{Y})
$$

and $[\boldsymbol{X}, \boldsymbol{Y}] \in \Gamma\left(D^{\prime}\right)$ if and only if the distribution $D_{1}$ is integrable.

Proof. The proof of this conclusion is done using the following equations:

$$
\begin{aligned}
& \mathrm{g}\left(\mathrm{w}^{2}[\boldsymbol{X}, \boldsymbol{Y}], \boldsymbol{Z}\right)=\sin ^{2} \theta \mathrm{g}([\boldsymbol{X}, \boldsymbol{Y}], \boldsymbol{Z})=0 \text { for } p>q, \\
& \mathrm{~g}\left(\mathrm{w}^{2}[\boldsymbol{X}, \boldsymbol{Y}], \boldsymbol{Z}\right)=\sinh ^{2} \theta \mathrm{g}([\boldsymbol{X}, \boldsymbol{Y}], \boldsymbol{Z})=0 \text { for } p<q,
\end{aligned}
$$

where $\boldsymbol{X}, \boldsymbol{Y} \in \Gamma\left(D_{1}\right), \boldsymbol{Z} \in \Gamma\left(D_{2}\right)$ and $\theta$ is a slant angle of the distribution $D_{2}$. So, the proof is easily completed.

Theorem 3.18. Let $\bar{M}$ be a semi-Riemannian submanifold of locally product semi-Riemannian manifold $M$. For any $\boldsymbol{X}, \boldsymbol{Y} \in \Gamma\left(D_{2}\right)$,

$$
\mathrm{P}_{1}\left(\nabla_{\boldsymbol{X}} \mathrm{fQ}_{2} \boldsymbol{Y}-\nabla_{\boldsymbol{Y}} \mathrm{fQ}_{2} \boldsymbol{X}\right)=\mathrm{P}_{1}\left(A_{\mathrm{w} \boldsymbol{X}} \boldsymbol{X}-A_{\mathrm{w} \boldsymbol{X}} \boldsymbol{Y}\right)
$$


if the distribution $D_{2}$ is integrable.

Proof. This proof is easily done with the help of Gauss and Weingarten equation.

Definition 3.19. Let $\bar{M}$ is an immersed lightlike submanifold of almost product semiRiemannian manifold $M$. Then, $\bar{M}$ is called a semi-slant lightlike submanifold if it provides the following conditions.

(i) $T \bar{M}=\operatorname{RadT} \bar{M} \perp\left(\mathrm{FRadT} \bar{M} \oplus \mathrm{Fltr} T \bar{M} \perp D^{\prime \prime}\right)$ where $D^{\prime \prime}$ has orthogonal direct decomposition $D^{\prime \prime}=D_{1} \oplus D_{2}$ and $D^{\prime \prime}$ is semi-Riemannian. Here, $D_{1}$ and $D_{2}$ are the distributions on $\bar{M}$.

(ii) $D_{1}$ is the invariant distribution that is $F D_{1}=D_{1}$

(iii) $D_{2}$ is the slant distribution with the slant angle $\theta$.

Because of the expressions and the proofs of the theorems which are given for semi-slant semiRiemannian submanifolds are similarly to this case, we omit them.

\section{References}

Aydın, G., and Çöken, A. C., 2013. "Slant submanifolds of semi-Riemannian manifolds. International Journal of Geometric Methods in Modern Physics, 10(10), 1350058.

Chen, B. Y., 1990. “Geometry of Slant Submanifolds”. Katholieke Universiteit, Leuven.

Duggal, L. K., and Bejancu, A., 1996. "Lightlike Submanifolds of Semi-Riemannian Manifolds and Applications”. Kluwer Academic Publishers, The Netherlands.

Li, H., and Liu, X., 2010. "Semi-slant submanifolds of a locally product manifold”. Georgian Mathematical Journal, 12(2), 273.

Şahin, B., 2006. “Slant submanifolds of an almost product Riemannian manifold”. J. Korean Math. Soc., 43(4), 717.

Şahin, B., 2008. "Slant lightlike submanifolds of indefinite Hermitian manifolds". Balkan Journal of Geometry and Its Applications, 13(1), 107.

Yano, K., and Kon, M., 1984. "Structures on Manifolds”. World Scientific Publishing Co. Pte. Ltd., Singapore. 